\section{P372 BACTERIAL VAGINOSIS AND HIGH-RISK HUMAN PAPILLOMAVIRUS COINFECTION AMONG AFRICAN AMERICAN WOMEN IN THE UNITED STATES}

${ }^{1}$ Purnima Madhivanan*, ${ }^{1}$ Makella Coudray, ${ }^{2}$ Daniel Ruiz-Perez, ${ }^{3}$ Brett Colbert, ${ }^{4}$ Karl Krupp ${ }^{5}$ Hansi Kumari, ${ }^{5}$ Kalai Mathee, ${ }^{2}$ Giri Narasimhan. ${ }^{1}$ Florida International University, Epidemiology, Miami, USA; ${ }^{2}$ Florida International University, Bioinformatics Research Group, Miami, USA; ${ }^{3}$ Florida International University, Department of Biological Sciences, College of Arts and Sciences, Miami, USA; ${ }^{4}$ Florida International University, Department of Health Promotion and Disease Prevention, Robert Stempel College of Public Health, Miami, USA; ${ }^{5}$ Florida International University, Biomolecular Sciences Institute, Miami, USA

\subsection{6/sextrans-2019-sti.474}

Background Bacterial vaginosis (BV) increases the risk of many sexually transmitted infections. The co-occurrence of persistent BV and high-risk HPV (HrHPV) increases the risk of developing cervical cancer. This study aims to investigate the cooccurrence of HrHPV and BV among young women in the US.

Methods Stored vaginal swabs were acquired from a previously completed clinical trial. The kinds of bacteria present in the samples were identified by classifying $16 \mathrm{~S}$ rRNA gene sequences in each sample using high-throughput pyrosequencing. HPV genotyping was performed using quantitative polymerase chain reaction using TaqMan probes in a customized plate (ThermoFisher Scientific; Waltham, Massachusetts). BV was classified using Nugent Scores of Gram Stain.

Results Eighty reproductive age African American (AA) women were included in the analysis. The point prevalence of HrHPV was $48.1 \%$ (95\%CI: 37-59\%). The mean age of the participants was 21.4 years (SD: 2.11), 81.2\% graduated high school. Prior antibiotic use was low (3.8\%), and $75 \%$ were not treated for BV during their lifetime. Among those who had been treated previously for BV, most women were treated $\geq$ five times (60\%). According to Nugent Scores, 70\% had BV, $13.7 \%$ had intermediate flora and $16.3 \%$ were healthy. Among HrHPV positive women, $66.7 \%$ were infected with single HrHPV genotype, 33.3\% with multiple HrHPV genotypes. Concurrent HrHPV and BV infection was found among $33.3 \%$ of the sample. However, there was no significant difference between the prevalence of HrHPV among women with and without BV.

Conclusion Co-occurence of hrHPV and BV among this group of young African American women was relatively high. Considering that these conditions are very common among women worldwide, further research in this field is imperative. More studies are needed to accurately evaluate temporal sequence of acquisition of both conditions in any attempt to establish a causal relationship.

Disclosure No significant relationships.

\section{P373 CO-OCCURRENCE OF BACTERIAL VAGINOSIS AND TRICHOMONAS VAGINALIS AMONG YOUNG AFRICAN AMERICAN WOMEN}

${ }^{1}$ Purnima Madhivanan*, ${ }^{1}$ Makella Coudray, ${ }^{2}$ Daniel Ruiz-Perez, ${ }^{3}$ Brett Colbert, ${ }^{4}$ Karl Krupp, ${ }^{5}$ Hansi Kumar, ${ }^{2}$ Giri Narasimhan, ${ }^{5}$ Kalai Mathee. ${ }^{1}$ Florida International University, Epidemiology, Miami, USA; ${ }^{2}$ Florida International University, Bioinformatics Research Group, Miami, USA; ${ }^{3}$ Florida International University, Department of Biological Sciences, College of Arts and Sciences, Miami, USA; ${ }^{4}$ Florida International University, Department of Health Promotion and Disease Prevention, Robert Stempel College of Public Health, Miami, USA; ${ }^{5}$ Florida International University, Biomolecular Sciences Institute, Miami, USA

10.1136/sextrans-2019-sti.475
Background While the etiology of bacterial vaginosis (BV) is still not known, it is described as a polymicrobial condition that lacks lactic-acid producing Lactobacillus species with an overgrowth of anaerobic bacteria and elevated vaginal $\mathrm{pH}$. This study aims to evaluate the relationship between BV assessed by Nugent scoring of vaginal Gram stain and Trichomonas vaginalis infection among African American young women in the U.S.

Methods Stored vaginal swabs from a previously completed clinical trial were acquired for this study. The kinds of bacteria present in the samples were identified by classifying $16 \mathrm{~S}$ rRNA gene sequences using high-throughput pyrosequencing. Vaginal smears were also categorized by the Nugent Gram stain score (0-3, normal; 4-6, intermediate state; 7-10, BV). TV genotyping was performed using quantitative polymerase chain reaction,performed using TaqMan probes in a customized plate (Thermo Fisher Scientific; Waltham, Massachusetts). Descriptive statistics were conducted to determine the odds of TV infection among women with BV.

Results This study included 80 African American reproductive age women with a mean age of 21.4 years (SD: 2.11 years). Most (81.2\%) women had graduated high school. 70\% (95\% CI: $37-59 \%$ ) had BV, $13.7 \%$ had intermediate and $16.3 \%$ had healthy vaginal flora. TV was diagnosed among 11.1\% (95\% CI: $4-8 \%)$ of the women. Prior antibiotic use was low $(3.8 \%)$, and $75 \%$ of women had not been treated for BV during their lifetime. Among those who were previously treated for BV, $60 \%$ were treated five or more time. Douching was reported by $49 \%$ of the sample. $55 \%$ of TV cases had concurrent BV, while $11.1 \%$ of TV cases also had intermediate vaginal flora. There were no association with prior antibiotic use, hormonal contraception, douching or prior treatment.

Conclusion Young African American women of reproductive age found to have abnormal vaginal flora should be screened for Trichomonas vaginalis infection.

Disclosure

No significant relationships.

\section{P374 OVERLAP BETWEEN AMSEL'S CRITERIA, NUGENT'S GRAM STAIN SCORE, AND VAGINAL MICROBIOTA COMMUNITY STATE TYPES}

${ }^{1}$ Antonio Salas, ${ }^{1}$ Khalil Ghanem, ${ }^{2}$ Rebecca Brotman, ${ }^{2}$ Jacques Ravel, ${ }^{1}$ Susan Tuddenham. 'Johns Hopkins University School of Medicine, Baltimore, USA; ${ }^{2}$ University of Maryland, Institute of Genome Sciences, Baltimore, USA

\subsection{6/sextrans-2019-sti.476}

Background Bacterial vaginosis (BV) is characterized by lowLactobacilli and increased anaerobes. BV can be determined by clinical assessment (Amsel's criteria) or microscopy (Nugent scoring); molecular methods are also under study. We investigated concordance between Amsel-BV, Nugent-BV and lowLactobacillus vaginal microbiota identified via $16 \mathrm{~S}$ rRNA gene sequencing.

Methods Vaginal swabs and clinical data were collected from women enrolled in a longitudinal study. Amsel's criteria were determined clinically and Nugent scoring (Nugent-BV=7-10, intermediate $=4-6$ ) was determined by microscopy. Vaginal microbiota were characterized using $16 \mathrm{~S}$ rRNA gene sequencing and categorized into 7 community state types (CSTs): 4 dominated by Lactobacillus spp. (CST I, II, III and V), and 3 by Streptococcus spp. (CST VI), Bifidobacterium spp. (CST VII), or a variety of anaerobes (CST IV). 
Results 110 samples, $\sim 10$ from each CST, were selected. $5.5 \% \quad(\mathrm{n}=6)$ had Amsel-BV, 32.7\% $(\mathrm{n}=36)$ Nugent-BV, and $36.4 \%(\mathrm{n}=40)$ had low-Lactobacillus CSTs (IV, VI, VII); $8.2 \%$ had symptoms. Among Amsel-BV samples, 83.3\% had NugentBV, $16.7 \%$ had intermediate Nugent score and all were CST IV. $86.1 \%$ of women with Nugent-BV and $85 \%$ of women with low-Lactobacillus CSTs did not have Amsel-BV. 22.2\% of those with Nugent-BV did not have low-Lactobacillus CSTs; of these 50\% were CST III (L. iners-dominated). 46.7\% of CST-III had a vaginal $\mathrm{pH} \geq 4.5$, and $13.3 \%$ had a Nugent BV score. 22 samples had a vaginal $\mathrm{pH} \geq 4.5$ and a normal Nugent score: $45.5 \%$ were in CST III, 9.1\% in low-Lactobacillus CSTs. $30.6 \%$ women with Nugent-BV had a vaginal $\mathrm{pH}$ $<4.5$; of these $45.5 \%$ were in CST IV.

Conclusion Nugent score and low-Lactobacillus CST were concordant. L. iners-dominated CSTs often had normal Nugent scores and high $\mathrm{pH}$. Among mostly asymptomatic women, a large proportion with low-Lactobacillus CSTs did not have Amsel-BV. Future studies assessing long term clinical outcomes will be needed to determine whether molecular methods provide added actionable or prognostic information.

Disclosure No significant relationships.

\section{P375 RISK FACTORS FOR INCIDENT BACTERIAL VAGINOSIS AMONG HETEROSEXUAL WOMEN}

${ }^{1}$ Christina Muzny ${ }^{*},{ }^{2}$ Kristal Aaron, ${ }^{1}$ Angela Pontius, ${ }^{1}$ Cheri Aycock, ${ }^{1}$ Jane Schwebke. ${ }^{1}$ University of Alabama at Birmingham, Division of Infectious Diseases, Birmingham, USA; ${ }^{2}$ University of Alabama at Birmingham, Medicine/Infectious Diseases, Birmingham, USA

\subsection{6/sextrans-2019-sti.477}

Background The exact etiology of bacterial vaginosis (BV) is unknown and it is controversial whether BV is a sexually transmitted infection (STI). Gardnerella vaginalis is present in $95-100 \%$ of BV cases. However, it is also found in women with normal vaginal flora and colonization is not sufficient for BV development. We investigated risk factors for incident BV (iBV) in a longitudinal study of heterosexual women.

Methods Women ages 18-45 with no Amsel criteria, Nugent score 0-3, no recent antibiotic use, and no evidence of STI were followed for iBV (Nugent score 7-10 on 3 consecutive days) with daily self-collected vaginal swabs for 8 weeks. Vaginal swabs were also obtained at enrollment for $G$. vaginalisquantitative PCR. Time to iBV was estimated and baseline participant characteristics were compared according to development of iBV or not. All analyses were conducted using SAS v9.4.

Results Between January 2015-May 2018, 164 eligible heterosexual women were enrolled, of which the majority were African American (73\%). Of those enrolled, 29 (17.7\%) developed iBV at a median time of 21 (range 6-50) days. Women with iBV were significantly more likely to be older $(33 \pm 7$ years vs $30 \pm 7$ years; $p=0.02)$, African American $(93 \%$ vs $69 \% ; \mathrm{p}=0.02)$, have a BV history $(72.4 \%$ vs $48.2 \%$; $\mathrm{p}=0.02)$, and have a pregnancy history $(78 \%$ vs $54 \%$; $\mathrm{p}=0.02)$. They were also significantly more likely to have detectable $G$. vaginalis by quantitative PCR at enrollment $(81 \%$ vs $60 \% ; \mathrm{p}=0.04)$ with a higher median number of copies/ $\mu \mathrm{L} \quad(4,850 \quad[1,890-30,100] \quad$ vs $1,340 \quad[217-9,550]$; $\mathrm{p}=0.03)$. No significant difference was found between women with iBV and those without regarding current tobacco use, history of bacterial STI, and lifetime number of sexual partners. Conclusion This study confirms previous cross-sectional findings of risk factors for $\mathrm{iBV}$ among heterosexual women and examines the potential role of $G$. vaginalis in $\mathrm{iBV}$ pathogenesis.

Disclosure No significant relationships.

\section{P380 THE ASSOCIATION BETWEEN SOCIAL SUPPORT, VIOLENCE EXPERIENCE, AND SOCIAL SERVICE NEEDS AMONG A SELECT SAMPLE OF URBAN ADULTS}

${ }^{1}$ Aruna Chandran*, ${ }^{2}$ Amanda Long, ${ }^{3}$ Yvonne Kingon, ${ }^{2}$ Jeannie Murray, ${ }^{4}$ Errol Fields, ${ }^{4}$ Christina Schumacher, ${ }^{3}$ Adena Greenbaum, ${ }^{2}$ Jacky Jennings. ${ }^{1} J o h n s$ Hopkins Bloomberg School of Public Health, Epidemiology, Baltimore, USA; ${ }^{2} J o h n s$ Hopkins University School of Medicine, Center for Child and Community Health Research (CCHR), Baltimore, USA; ${ }^{3}$ Baltimore City Health Department, Baltimore, USA; ${ }^{4}$ Johns Hopkins School of Medicine, Baltimore, USA

\subsection{6/sextrans-2019-sti.478}

Background Experiencing violence is associated with negative health outcomes including increased substance use, mental health problems, and sexual risk behaviors. Studies show associations between strong perceived social support and decreased negative health outcomes. The objectives of this study were: (1) determine the impact of violence (exposure and victimization) on social service needs and perceived health status, and (2) assess whether social support is associated with decreased violence experience among a select sample of urban adults.

Methods A cross-sectional survey was conducted among 187 adults seen in one of 7 urban clinics as part of a larger project between September 2015 and March 2018. A multivariable logistic regression model was used, adjusting for the clinic site as well as age and gender identity.

Results 131 (72\%) individuals had ever witnessed and 89 (49\%) had ever experienced physical assault. Violence victimization was associated with an 8.6-fold odds (95\% CI: 2.4, 31.4) of needing housing assistance and 3.4-fold increased odds (95\% CI: $1.4,8.5$ ) of needing mental health/substance use support services. Exposure to violence was associated with a 5.2-fold increased odds (95\% CI: 2.0, 13.4) of needing housing assistance, a 12.4-fold increased odds (95\% CI: 2.1, 74.0) of needing transportation support, and a 0.5 decreased odds (95\% CI: $0.3,0.8$ ) of reporting optimal health status. Increased perceived social support was associated with 0.54 odds (95\% CI: $0.4,0.8$ ) and 0.62 odds (95\%CI: $0.4,0.9)$ of violence exposure and victimization, respectively.

Conclusion Violence experiences were common in this sample of urban adults, and were associated with increased social service needs and suboptimal perceived health status. Augmenting sources of social support is one possible mechanism for addressing these issues. Including care for the effects of experiencing violence as well as social service needs are important in optimizing the health of key populations.

Disclosure No significant relationships. 